\title{
Evaluation of pentraxin-3 in familial Mediterranean fever patients during attack and attack-free periods
}

\author{
Gok $\mathrm{M}^{1}$, Sirkeci $\mathrm{O}^{2}$, Kara $\mathrm{M}^{3}$, Sakin $\mathrm{YS}^{4}$, Tanoglu $\mathrm{A}^{3}$, Sirkeci EE ${ }^{5}$, Oztin $\mathrm{H}^{6}$, Duzenli $\mathrm{T}^{3}$, \\ Kaplan $\mathrm{M}^{3}$, Yazgan $\mathrm{Y}^{3}$, Ipcioglu $\mathrm{OM}^{7}$
}

Sultan Abdulhamid Han Training and Research Hospital, Department of Gastroenterology, Istanbul, Turkey. tolgaduzenli@yahoo.com

\begin{abstract}
INTRODUCTION: Pentraxin-3 (PTX-3) is a prototype of pentraxin proteins that have been shown to be involved in acute phase response. In this study, we aimed to investigate the relationship between PTX-3 levels and familial Mediterranean fever (FMF) disease, and to evaluate PTX-3 as a novel diagnostic marker of FMF. METHOD: Forty-three male patients diagnosed with FMF and 42 healthy individuals were included in the study. Patients with other inflammatory diseases and patients who used drugs having anti-inflammatory properties were excluded from the research. Blood samples were obtained during both attack and attack-free periods. RESULTS: Patient attack periods were confirmed by combining physical examination and elevation of acute phase reactants. Acute phase reactants were significantly higher in attack versus attack-free periods $(p<0.01)$, however PTX-3 levels were not significantly different between the two periods. Additionally, PTX-3 levels in FMF patients were higher than in controls in both attack $(917.29 \pm 725.29$ vs $451.83 \pm 291.95, p<0.01)$ and attackfree periods ( $748.23 \pm 487.53$ vs $451.83 \pm 291.95, p<0.01)$.

CONCLUSION: In this study, we showed that PTX-3 levels, in both FMF attack and attack-free periods, were significantly higher than in the control group. Finally, PTX-3 may be a promising biomarker for FMF diagnosis and may predict FMF attacks (Tab. 2, Fig. 2, Ref. 18). Text in PDF www.elis.sk.

KEY WORDS: familial Mediterranean fever, pentraxin-3, inflammation.
\end{abstract}

\section{Introduction}

Familial Mediterranean fever (FMF) is defined by repetitive periods of fever, inflammation and pain in chest, abdomen or joints and with additional findings such as myalgia and erysipelas-like skin lesions throughout life. Although FMF has a distinctly ethnic distribution, FMF patients have been reported in many countries all around the world. FMF is especially seen in the Mediterranean region in Sephardic Jews, Armenians, Turks, Greeks, Arabs and Italians (1-4). It has been shown that there is a mutation in chromosome 16 in the Mediterranean fever (MEFV) gene (5). This gene encodes pyrin protein which has an important role in inflammation and apoptosis (6). As a result of the MEFV mutation, pyrin and

${ }^{1}$ Department of Internal Medicine, Sultan Abdulhamid Han Training and Research Hospital, Istanbul, Turkey, ${ }^{2}$ Department of Internal Medicine, Near East University Hospital, Lefkoşa, Turkish Republic of Northern Cyprus, ${ }^{3}$ Department of Gastroenterology, Sultan Abdulhamid Han Training and Research Hospital, Istanbul, Turkey, ${ }^{4}$ Department of Gastroenterology, Gulhane Training and Research Hospital, Ankara, Turkey, ${ }^{5}$ Department of Emergency Medicine, Near East University Hospital, Lefkoşa, Turkish Republic of Northern Cyprus, ${ }^{6}$ Department of Geriatrics, Gulhane Training and Research Hospital, Ankara, Turkey, and ${ }^{7}$ Department of Biochemistry, Sultan Abdulhamid Han Training and Research Hospital, Istanbul, Turkey

Address for correspondence: T. Duzenli, MD, Sultan Abdulhamid Han Training and Research Hospital, Department of Gastroenterology, Tibbiye Caddesi Uskudar, 34688, Istanbul, Turkey.

Phone: +90.216 .5423502 , Fax: +90.216 .5422192$ anti-inflammatory protein production decreases and inflammatory conditions cannot be suppressed, thereby resulting in fever and inflammation at certain locations of the body.

Currently, there is no definitive technique for the diagnosis of FMF disease and the diagnosis is made by monitoring for clinical signs according to the criteria defined by Tel Hashomer (2-4). Although not necessary, detecting a mutation in MEFV (Mediterranean fever) gene favors the diagnosis in patients who have clinical findings. The other finding that supports the diagnosis is that of elevated blood levels of acute phase reactants at the attack periods. It was found that there is an excessive activation of cytokine cascades during the disease (5-8). Both attack-free and attack periods show a significant increase in proinflammatory cytokines. Abnormal pyrin protein that occurs as a result of MEFV gene mutations makes the blocking suppression of inflammation ineffective and is thought to be responsible for this ongoing inflammatory process (9).

Pentraxin-3 (PTX-3) is an acute phase reactant located in the long pentraxin group. PTX-3 is produced as a result of inflammatory cytokines such as TLR (toll-like receptor) agonists, IL-1 $\beta$ and tumor necrosis factor-alpha (TNF- $\alpha$ ). There are abundant cells that play a role in PTX-3 production, such as dendritic cells, monocytes, macrophages, vascular endothelial cells, kidney epithelial cells, fibroblasts, adipocytes, smooth muscle cells, glial cells, mesangial cells and synovial cells (10). Inflammation has been shown to be associated with increased levels of serum PTX-3 in several studies (11-13). Our aim was to investigate the levels of 
PTX-3 among FMF patients, and to evaluate PTX-3 as a novel diagnostic marker of FMF.

\section{Materials and methods}

In this study, 43 male patients who were admitted to our department and diagnosed with FMF between November 2013 and March 2014 were accepted as the patient group and 42 healthy male individuals who had similar characteristics and did not have any active or chronic disease were accepted as the control group. This study was authorized by our hospital's medical ethics committee. Informed consents were signed by all subjects in this study.

FMF attack period was diagnosed by combining a positive physical examination with high levels of acute phase reactants (APR). All pain locations were examined and noted for further evaluation and classification. During both FMF attack-free and attack periods, physical examination was performed, and blood samples were taken in order to evaluate APR levels (erythrocyte sedimentation rate (ESR), fibrinogen and leucocyte count). Blood samples were obtained in the first 24 hours of the attack period, and at least 10 days after the last FMF attack in an attack-free period. Patients who used nonsteroidal anti-inflammatory drugs, steroids, immune suppressed or immune-modulating drugs and patients who had chronic diseases such as cardiovascular disease, acute or chronic liver or kidney disease, chronic obstructive lung disease, rheumatologic disease, malignancy, thyroid disease, immune deficiency, hypertension, diabetes mellitus, acute/chronic infection, bleeding disease and those who did not completely fulfill the criteria for research were excluded from the study.

Blood samples were centrifuged for 5 minutes at $5000 \mathrm{rpm}$ and a volume of $2 \mathrm{cc}$ of serum was separated into Eppendorf tubes for PTX-3 studies and stored at $-80^{\circ} \mathrm{C}$. After reaching a sufficient sample size, all samples were analyzed in the biochemistry lab.

ESR was examined with a Vacuet at Sed Rate Screener 100 device by precipitation, fibrinogen was examined with an Architech ci8200 device, and complete blood count was examined with a Cell-Dyn Sapphire device. PTX-3 was examined with a Booster Elx 800 Instruments- Biotek Biotek Plate Reader and Elpan-40 by ELISA using an Instruments- Plate Washer.

\section{Statistical analysis}

The research was designed as an observational, prospective, case-control study. SPSS 15.0 was used for statistical analysis. The average value of the parameters in the patient and control

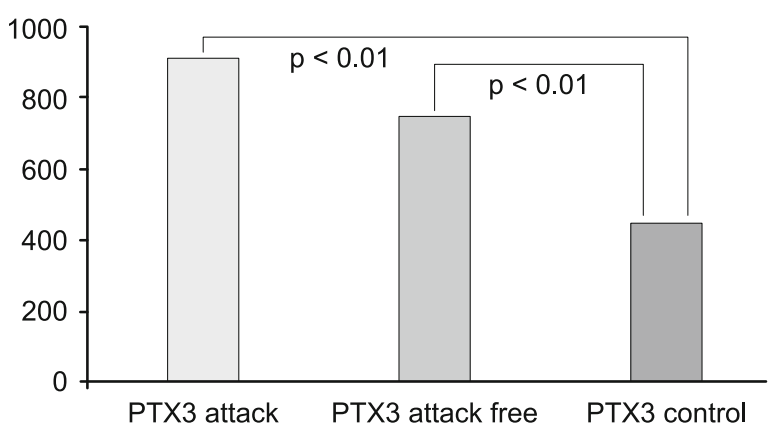

Fig. 1. Comparison of PTX 3 levels between FMF patients and healthy controls according to attack period. The PTX 3 levels in both attack period and attack free period.were significantly higher than in control group.

groups, range of values and standard deviations were calculated, and Kolmogorov-Smirnov test was used for compliance of the data with normal distribution. Student T-test and Mann-Whitney U test were used for comparisons. Roc analysis was used to evaluate the sensitivity and specificity for PTX-3 in patients with FMF. Pearson analysis was used for correlations. $\mathrm{p}<0.05$ was assessed as statistically significant.

\section{Results}

Forty-three male FMF patients with attack periods and 42 healthy controls were included in the study. The control group consisted of healthy volunteers of similar age and gender. The data of all groups are shown in Table 1.

When data from patients with FMF attacks and asymptomatic periods were compared, APRs were higher in the attack period $(\mathrm{p}<0.01)$ (Tab. 1). PTX-3 levels in the attack group were higher than in the asymptomatic period, but this difference was not statistically significant $(\mathrm{p}>0.05)$.

White blood cell, ESR, fibrinogen and PTX-3 levels in the attack period in FMF patients were significantly higher than in the control group (Fig. 1).

Although ESR and fibrinogen were within normal values, they were significantly higher in the attack-free group compared to the control group. Additionally, PTX-3 levels were significantly higher in the asymptomatic period $(748.23 \pm 487.53$ vs $451.83 \pm$ 291.95; $\mathrm{p}<0.01)$. No differences were found in terms of age and white blood cell count $(\mathrm{p}>0.05)$.

Tab. 1. Data of patient groups. Reactant levels in attack period were significantly higher than in both attack-free period and control group. Pentraxin-3 (PTX-3) level in attack period was significantly higher when compared to that in control group, but not significantly higher when compared to that in asymptomatic period.

\begin{tabular}{|c|c|c|c|}
\hline \multirow[t]{2}{*}{ Parameters } & \multicolumn{3}{|c|}{ Patient group } \\
\hline & Attack Period $(n=43)$ & Attack-free Period $(n=43)$ & Control Group $(n=42)$ \\
\hline Erythrocyte Sedimentation Rate $(\mathrm{mm} / \mathrm{h})$ & $42.00 \pm 45.45^{\mathrm{a}, \mathrm{b}}$ & $5.95 \pm 4.81^{\mathrm{c}}$ & 4,09 \\
\hline Fibrinogen $(\mathrm{mg} / \mathrm{dl})$ & $488.55 \pm 94.71^{\mathrm{a}, \mathrm{b}}$ & $277.79 \pm 63.63^{c}$ & $262.81 \pm 52.10$ \\
\hline Age & $21.81 \pm 3.40$ & $21.81 \pm 3.40$ & $21.86 \pm 3.57$ \\
\hline Leucocyte $\left(\times 10^{3} / \mathrm{mm}^{3}\right)$ & $13516.67 \pm 2800.8^{\mathrm{a}, \mathrm{b}}$ & $6502.38 \pm 2116.5$ & $6809.52 \pm 2011.1$ \\
\hline PTX-3 (pg/ml) & $917.29 \pm 725.29^{a}$ & $748.23 \pm 487.53^{\mathrm{c}}$ & $451.83 \pm 291.95$ \\
\hline
\end{tabular}

${ }^{a}=\mathrm{p}<0.01$ vs control group; ${ }^{b}=\mathrm{p}<0.01$ vs asymptomatic period; ${ }^{\mathrm{c}}=\mathrm{p}<0.01$ vs control group 
490-493

Tab. 2. Overall accuracy and ROC analysis of PTX-3 among patients with FMF.

\begin{tabular}{|c|c|c|c|c|c|c|c|}
\hline & & Cut-off value & Sensitivity & Specificity & PPV (\%) & NPV (\%) & Overall accuracy (\%) \\
\hline \multirow{2}{*}{ PTX-3 } & Attack period & 721.5 & 92.9 & 58 & 89.3 & 68.4 & 75.2 \\
\hline & Asymptomatic period & 696.2 & 90 & 54 & 85.2 & 65.5 & 71.6 \\
\hline
\end{tabular}

PTX-3 - pentraxin-3; PPV - positive predictive value; NPV - negative predictive value

The sensitivity, specificity, positive and negative predictive values of PTX-3 in acute attack and asymptomatic periods are shown in Table 2, and ROC curves of attack and asymptomatic periods are shown in Figure 2. When a PTX-3 level cut-off value of 696.2 was used, the test's positive predictive value was $85.2 \%$ with $71.6 \%$ overall accuracy (Tab. 2). According to correlation analysis, there was no correlation between white blood cell count, fibrinogen, ESR and PTX.

\section{Discussion}

In this study, we evaluated the association between PTX-3 levels and other inflammation markers in FMF patients during both symptomatic and asymptomatic periods. We showed that PTX-3 levels were significantly higher in FMF patients than in healthy individuals, regardless of attack period.

During the attack period in FMF disease, higher levels of ESR, fibrinogen and white blood cell count when compared to an attackfree period is an expected result and has been shown in many studies $(10,14)$. Similarly, we found elevated APRs in the FMF attack period. As opposed to these studies, we found ESR, fibrinogen and leukocyte levels to be significantly higher in the asymptomatic period than in the control group. Nevertheless, these parameters were also within normal levels in the asymptomatic period. Therefore, we think that this significance might not be important. In addition to these findings, Bulut et al (14) demonstrated elevated PTX-3 levels in FMF patients when compared with healthy controls. We found similar findings in our study. Interestingly, PTX-3 levels were also higher in asymptomatic period when compared with the control group, while this elevation in attack period did not differ from that in attack-free period. Therefore, these findings may be a clue to PTX-3 as a diagnostic laboratory test for suspected FMF.

PTX-3 is one of the major acute phase reactants, and in inflammatory conditions serum levels can increase up to 3-5 times from the basal value. PTX-3 is produced as a result of inflammatory cytokines such as TLR (toll-like receptor), IL- $1 \beta$ and TNF- $\alpha$. PTX-3 is stored in specific granules in neutrophils and is released into the blood as a result of stimulation of TLRs. In addition, IL$1 \beta$ and IL- 6 stimulate the release of large amounts of PTX-3 from peripheral mononuclear cells (11).
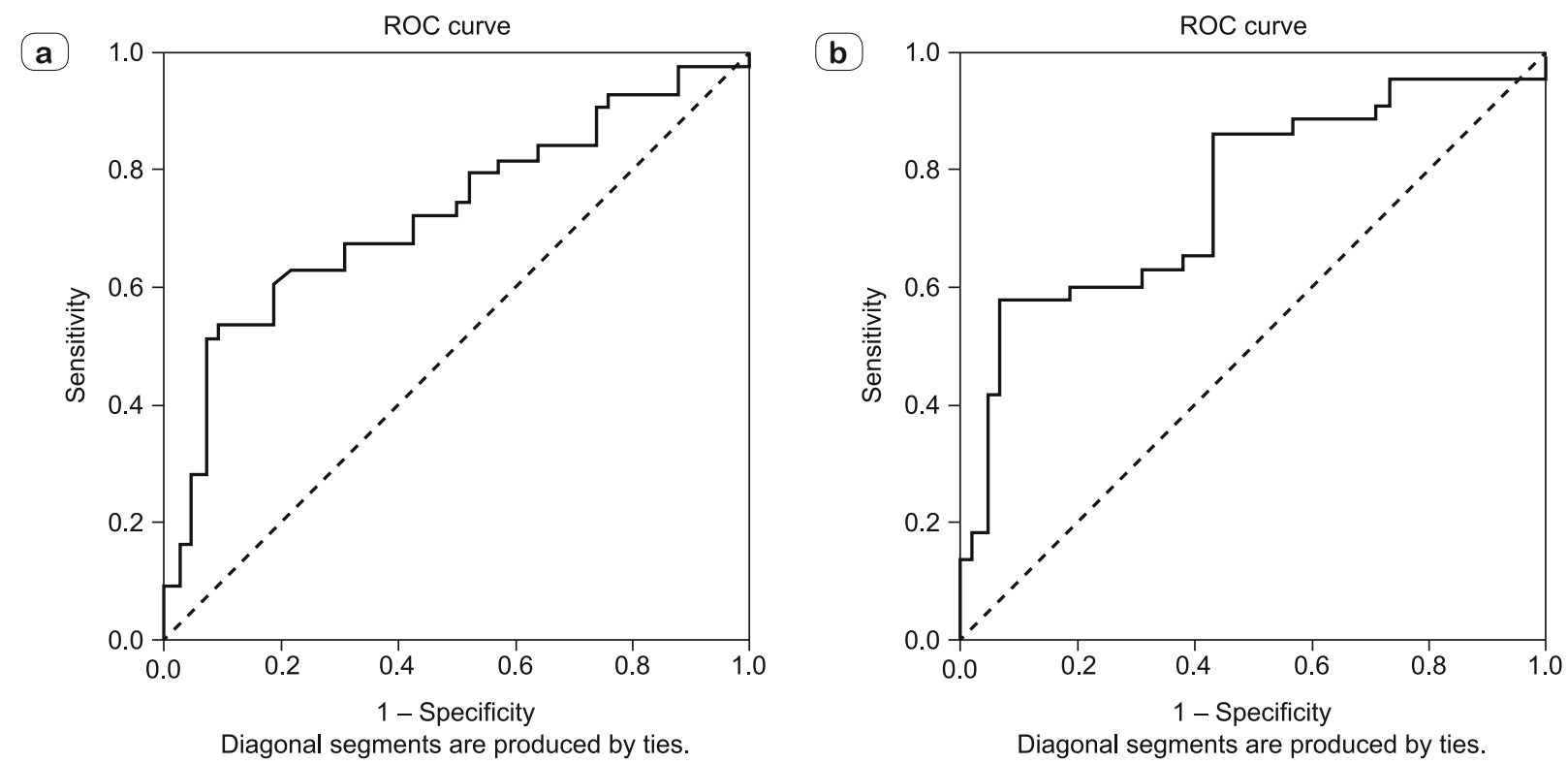

Diagonal segments are produced by ties.

\begin{tabular}{|c|c|c|c|c|}
\hline \multirow{2}{*}{ Area } & \multirow{2}{*}{ Std. error ${ }^{a}$} & \multirow{2}{*}{$\begin{array}{l}\text { Asymptotic } \\
\text { sig. }\end{array}$} & \multicolumn{2}{|c|}{$\begin{array}{l}\text { Asymptotic 95\% } \\
\text { Confidence interval }\end{array}$} \\
\hline & & & $\begin{array}{l}\text { Lower } \\
\text { bound }\end{array}$ & $\begin{array}{l}\text { Upper } \\
\text { bound }\end{array}$ \\
\hline 0.724 & 0.056 & 0.000 & 0.614 & 0.834 \\
\hline
\end{tabular}

\begin{tabular}{|c|c|c|c|c|}
\hline \multirow{2}{*}{ Area } & \multirow{2}{*}{ Std. error ${ }^{a}$} & \multirow{2}{*}{$\begin{array}{l}\text { Asymptotic } \\
\text { sig. }{ }^{\text {b }}\end{array}$} & \multicolumn{2}{|c|}{$\begin{array}{l}\text { Asymptotic } 95 \% \\
\text { Confidence interval }\end{array}$} \\
\hline & & & $\begin{array}{l}\text { Lower } \\
\text { bound }\end{array}$ & $\begin{array}{l}\text { Upper } \\
\text { bound }\end{array}$ \\
\hline 0.755 & 0.053 & 0.000 & 0.651 & 0.860 \\
\hline
\end{tabular}

Fig. 2. Reciever operating characteristic curve for pentraxin 3 for differentiating between FMF patients and healthy controls. (a) ROC characteristic between attack-free period and control; (b) ROC characteristic between attack period and control. 
Studies of PTX-3 pointed out that it increases in some inflammatory diseases $(12,13)$. Ishihara et al $(12)$ reported that serum PTX-3 levels were higher in the period of active disease of Takayasu arthritis when compared to both inactive period and a control group. Finally, they concluded that PTX-3 is more specific than CRP in demonstrating arterial inflammation. In a study investigating PTX-3 levels with forty-three patients with vasculitis, PTX-3 levels were higher in active vasculitis than in inactive period of vasculitis (13). In addition, PTX-3 levels were similar to those in healthy control group and inactive vasculitis group. As opposed to the latter findings and in accordance with Bulut et al (14), we found that serum PTX-3 levels were significantly higher in the asymptomatic period. This difference from other inflammatory conditions might be described as an ongoing subclinical inflammation in FMF disease. Thus, our findings may suggest that PTX-3 levels may be used as a diagnostic marker for FMF disease.

In this study, PTX-3 levels were absolutely higher in the attack period than in the attack-free period, but this result was not significant. First of all, colchicine treatment may cause this consequence since colchicine has anti-inflammatory effects and can cause a decrease in proinflammatory cytokine levels (15-17). Another reason may be in the cross-sectional design of the study and the results of samples reflect just one time point. PTX-3 serum levels do not have to reach peak levels at the time of blood test. Therefore, studies with a larger number of patients and with blood samples taken consecutively for 2 or 3 days of attack for PTX-3 measurement may give more accurate results.

One of the important results of our study was that PTX-3 levels were higher in attack or attack-free patients than in the healthy individuals. Studies have shown that also in attack-free periods, inflammation continues. Duzova et al (16) showed that in the attackfree period, subclinical inflammation continued in FMF patients and they stated that SAA may be a good marker for demonstrating subclinical inflammation. Haznedaroglu et al (18) showed that cytokines IL-17 and IL-18 remained high in the attack-free periods. Therefore, as with other acute phase reactants, high levels of PTX-3 may indicate that PTX-3 has a role in the pathogenesis of subclinical inflammation in FMF patients, and that PTX-3 may be used as a biomarker for the determination of inflammatory process in FMF.

Our study has some limitations. Firstly, the study population was not appreciably large. However, FMF is a rare disease, and it is not easy to include FMF patients in both periods. Secondly, all study participants were male, since the research was carried out in a tertiary military center.

In conclusion, we have shown that APRs were significantly higher in the attack period in accordance with current literature and that PTX-3 levels were significantly higher in FMF patients than in healthy controls regardless of the disease period. Thus, this difference may help in the diagnosis of FMF disease regardless of the attack period of the disease.

\section{References}

1. Oncu K, Yazgan Y, Tanoglu A et al. Can serum fetuin-A be regarded as an inflammatory marker among patients with familial Mediterranean fever? Dig Dis Sci 2013; 58 (11): 3212-3217.
2. Dinç M, Tanoğlu A, Yazgan Y et al. Serum matrix metalloproteinase-9 and tissue inhibitor of metalloproteinase-1 expression in patients with familial Mediterranean fever. Turk J Gastroenterol 2015; 26 (6): 487-491.

3. Toplak N, Frenkel J, Ozen $\mathbf{S}$ et al. An international registry on autoinflammatory diseases: the Eurofever experience. Ann Rheum Dis 2012; 71 (7): $1177-1182$.

4. Livneh A, Langevitz P. Diagnostic and treatment concerns in familial Mediterranean fever. Baillieres Best Pract Res Clin Rheumatol 2000; 14 (3): 477-498.

5. Moradian MM, Babikyan D, Banoian D et al. Comprehensive analysis of mutations in the MEFV gene reveal that the location and not the substitution type determines symptom severity in FMF. Mol Genet Genomic Med 2017; 5 (6): 742-750.

6. Cekin N, Akyurek ME, Pinarbasi E, Ozen F. MEFV mutations and their relation to major clinical symptoms of Familial Mediterranean Fever. Gene 2017 30; 626: 9-13.

7. Touitou I, Lesage S, McDermott $M$ et al. Infevers: an evolving mutation database for auto-inflammatory syndromes. Hum Mutat 2004; 24 (3): 194-198.

8. Touitou I, Milhavet F, Cuisset L. Response to Li and Zhang: infevers, a human gene mutation database for autoinflammatory diseases including disseminated superficial actinic porokeratosis. J Dermatol Sci 2014; 75 (3): 208-209.

9. Direskeneli H, Ozdogan H, Korkmaz C, Akoglu T, Yazici H. Serum soluble intercellular adhesion molecule 1 and interleukin 8 levels in familial Mediterranean fever. J Rheumatol 1999; 26 (9): 1983-1986.

10. Korkmaz C, Ozdogan H, Kasapçopur O, Yazici H. Acute phase response in familial Mediterranean fever. Ann Rheum Dis 2002; 61 (1): $79-81$.

11. Moalli F, Jaillon S, Inforzato A et al. Pathogen recognition by the long pentraxin PTX3. J Biomed Biotechnol 2011; 2011: 830421.

12. Ishihara T, Haraguchi G, Kamiishi T, Tezuka D, Inagaki H, Isobe M. Sensitive assessment of activity of Takayasu's arteritis by pentraxin3, a new biomarker. J Am Coll Cardiol 2011; 57 (16): 1712-1713.

13. Fazzini F, Peri G, Doni A et al. PTX3 in small-vessel vasculitides: an independent indicator of disease activity produced at sites of inflammation. Arthritis Rheum 2001; 44 (12): 2841-2850.

14. Bulut M, Ceri M, Unverdi S et al. Higher Pentraxin-3 Levels are Associated With Inflammation in Familial Mediterranean Fever. J Clin Lab Anal 2016; 30 (6): 978-981.

15. Notarnicola C, Didelot MN, Koné-Paut I, Seguret F, Demaille J, Touitou I. Reduced MEFV messenger RNA expression in patients with familial Mediterranean fever. Arthritis Rheum 2002; 46 (10): 2785-2793.

16. Duzova A, Bakkaloglu A, Besbas $N$ et al. Role of A-SAA in monitoring subclinical inflammation and in colchicine dosage in familial Mediterranean fever. Clin Exp Rheumatol 2003; 21 (4): 509-514.

17. Kiraz S, Ertenli I, Arici M et al. Effects of colchicine on inflammatory cytokines and selectins in familial Mediterranean fever. Clin Exp Rheumatol 1998; 16 (6): 721-724.

18. Haznedaroglu S, Oztürk MA, Sancak B et al. Serum interleukin 17 and interleukin 18 levels in familial Mediterranean fever. Clin Exp Rheumatol 2005; 23 (4 Suppl 38): S77-80. -

Received March 25, 2018. Accepted April 27, 2018. 